\title{
EDITORIAL
}

\section{Halide perovskites: from materials to optoelectronic devices}

\author{
Jiang TANG (ه), Dehui LI (ه) \\ School of Optical and Electronic Information, Wuhan National Laboratory for Optoelectronics, Huazhong University of Science and Technology, \\ Wuhan 430074, China
}

(C) Higher Education Press 2020

Halide perovskites have been extensively studied in last decade partially due to the unprecedentedly rapid increase of power conversion efficiency of perovskite based solar cells. In addition to the solar cells, perovskite based optoelectronic devices such as photodetectors and light emitting devices have also been demonstrated with impressive performance, benefited from the large absorption coefficient, tunable band gap, defect tolerance and long carrier diffusion length. Although significant progress has been made in these fields, a few challenges including long-term stability and toxicity of lead greatly limit their commercialization. Great effort has been put into those fields to address the long-last issues from aspects of fundamental understanding of their photophysics, material engineering and performance optimization. This special issue on "Halide perovskites: from materials to optoelectronic devices" including one comment, four reviews, and five original research articles covers all topics mentioned.

In this special issue, Xiong et al. [1] from Nanyang Technological University (Singapore) give an in-depth comment on the current development and future research direction of Bose-Einstein condensation of exciton polariton based on perovskites. Koleilat et al. [2] give a thorough summary on how the dimensionality engineering including morphological engineering and molecular engineering could affect their band gap, binding energy and carrier mobility and subsequently the performance of photodetectors and solar cells. Li et al. [3] review the research process of self-trapped excitons in two-dimensional perovskite including the origins of self-trapped excitons, how to detect and control the self-trapped excitons and how the presence of self-trapped excitons influences the performance of perovskite based optoelectronic devices. Tang et al. [4] collect the performance matrix including external quantum efficiency, luminance, and stability status of perovskite based light emitting diodes, presenting a brief yet comprehensive overview of this field to readers. Chen et al. [5] summarize the possible top cells for next-generation Si-based tandem solar cells and further propose the promising candidate top cells. Mei et al. [6] explore how the precursor concentrations affects the performance of printable hole-conductor-free mesoscopic perovskite solar cells via a simple one-step drop-coating method while You et al. [7] investigate the performance and thermal stability of inorganic perovskite solar cells by using dopant-free polymer, poly(3-hexylthiophene-2,5-diyl) (P3HT), as the holetransport layer. Zhong et al. [8] fabricate a wide-bandgap formamidinium lead bromide film using a doctor-bladecoating method and study the effect of the species of surfactants on the performance of the solar cells based on the as-fabricated film. Wei et al. [9] demonstrate how to fabricate efficient perovskite based light emitting diodes via composite engineering. Mu et al. [10] propose a corona modulation device structure to achieve random lasing in perovskite quantum dots under electron beam excitation.

These ten articles appeared in this special issue only cover a rather small portion of the recent advances in this rapid growing perovskite community. We hope this special issue will provide useful references for halide perovskite community and motivate more investigations in these research fields.

Received September 1, 2020

E-mails: jtang@mail.hust.edu.cn (J. Tang), dehuili@hust.edu.cn (D. Li) 


\section{References}

1. Wen X, Xiong Q. Bose-Einstein condensation of exciton polariton in perovskites semiconductors. Frontiers of Optoelectronics, 2020, 13(3): 193195

2. Kahwagi R F, Thornton S T, Smith B, Koleilat G I. Dimensionality engineering of metal halide perovskites. Frontiers of Optoelectronics, 2020, 13(3): 196-224

3. Li J, Wang H, Li D. Self-trapped excitons in two-dimensional perovskites. Frontiers of Optoelectronics, 2020, 13(3): 225-234

4. Du P, Gao L, Tang J. Focus on performance of perovskite light-emitting diodes. Frontiers of Optoelectronics, 2020, 13(3): 235-245

5. Lu S, Chen C, Tang J. Possible top cells for next-generation Si-based tandem solar cells. Frontiers of Optoelectronics, 2020, 13(3): 246-255

6. Jiang S, Sheng Y, Hu Y, Rong Y, Mei A, Han H. Influence of precursor concentration on printable mesoscopic perovskite solar cells. Frontiers of Optoelectronics, 2020, 13(3): 256-264

7. Mu S, Ye Q, Zhang X, Huang S, You J. Polymer hole-transport material improving thermal stability of inorganic perovskite solar cells. Frontiers of Optoelectronics, 2020, 13(3): 265-271

8. Ying H, Liu Y, Dou Y, Zhang J, Wu Z, Zhang Q, Cheng Y B, Zhong J. Surfactant-assisted doctor-blading-printed FAPbBr $\mathrm{F}_{3}$ films for efficient semitransparent perovskite solar cells. Frontiers of Optoelectronics, 2020, 13(3): 272-281

9. Yan C, Lin K, Lu J, Wei Z. Composition engineering to obtain efficient hybrid perovskite light-emitting diodes. Frontiers of Optoelectronics, 2020, 13(3): 282-290

10. Zhu Y, Mu Y, Tang F, Du P, Ren H. A corona modulation device structure and mechanism based on perovskite quantum dots random laser pumped using an electron beam. Frontiers of Optoelectronics, 2020, 13(3): 291-302

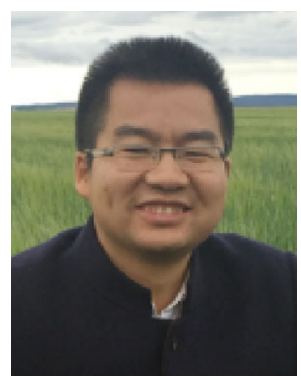

Jiang Tang is a professor at Wuhan National Laboratory for Optoelectronics, Huazhong University of Science and Technology (HUST), China. He obtained his Ph.D. degree from University of Toronto, Canada at 2010 and joined HUST in 2012. He has published $>150$ papers with $>11000$ times citation. His current research focuses on halide perovskites for X-ray detection and light emitting diodes, colloidal quantum dot infrared photodetectors, and antimony selenide thin film solar cells.

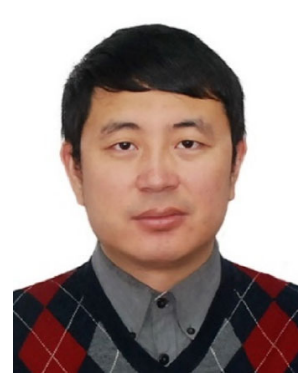

Dehui Li is a professor in School of Optical and Electronic Information at Huazhong University of Science and Technology in China. He obtained his Ph.D. degree from Nanyang Technological University, Singapore in 2013 and was a postdoctoral fellow with Prof. Xiangfeng Duan (2013-2016) at University of California, Los Angeles, USA. His current research interests include low dimensional halogen perovskites, two-dimensional layered materials and surface plasmons in optoelectronics field. He has published more than 70 papers in high-profile journals such as Nature, Nature Communications, Science Advances, Advanced Materials, Nano Letters, and ACS Nano. 